\title{
Ulrich bundles on some twisted flags
}

\author{
Saša Novaković
}

August 2018

\begin{abstract}
In this note we prove that certain twisted flag varieties carry Ulrich bundles.
Let $X \subset \mathbb{P}^{N}$ be a projective variety of dimension $d$. An Ulrich bundle on $X$ is a vector bundle $\mathcal{E}$ satisfying $H^{i}(X, \mathcal{E}(-l))=0$ for any $i \in \mathbb{Z}$ and $1 \leq l \leq d$. This notion was introduced in 13 , where the authors ask whether every projective variety admits an Ulrich bundle. The answer is known in a few cases: curves and Veronese varieties [13, [17, complete intersections [7, generic linear determinantal varieties [6], Segre varieties [10], rational normal scrolls 19, 15, Grassmannians [1, some flag varieties [11, 9], some isotropic Grassmannians [16], K3 surfaces [2], 14], abelian surfaces [3], Enriques surfaces [8], ruled surfaces [1] and Brauer-Severi varieties [21. We refer to 4] for an introduction to this subject. In this short note we prove that the existence of Ulrich bundles on certain twisted flags of type $A_{n}, B_{n}, C_{n}$ and $D_{n}$ follows easily from known results for partial flags and descent theory.
\end{abstract}

We recall the definition and some facts on twisted flags and refer to 20 for details. Let $G$ be a semisimple algebraic group over a field $k$ and $G_{s}=G \otimes_{k} k^{s e p}$. For a parabolic subgroup $P$ of $G_{s}$, one has a homogeneous variety $G_{s} / P$. A twisted flag is variety $X$ such that $X \otimes_{k} k^{\text {sep }}$ is $G_{s}$-isomorphic to $G_{s} / P$ for some $G$ and some parabolic $P$ in $G_{s}$. Any twisted flag is smooth, absolutely irreducible and reduced. An algebraic group $G^{\prime}$ is called twisted form of $G$ iff $G_{s}^{\prime} \simeq G_{s}$ iff $G^{\prime}={ }_{\gamma} G$ for some $\gamma \in Z^{1}\left(k, \operatorname{Aut}\left(G_{s}\right)\right)$. The group $G^{\prime}$ is called an inner form of $G$ if there is a $\delta \in Z^{1}\left(k, \bar{G}\left(k^{\text {sep }}\right)\right)$ with $G^{\prime}={ }_{\delta} G$. Here $\bar{G}=G / Z(G)$. For an arbitrary semisimple $G$ over $k$, there is a unique (up to isomorphism) split semisimple group $G^{d}$ such that $G_{s} \simeq G_{s}^{d}$. If $G$ is an inner form of $G^{d}$, then $G$ is said to be of inner type. For instance, let $A$ be a central simple algebra over $k$ of degree $n$ and $G=\mathrm{PGL}_{1}(A)$, then $G_{s} \simeq \mathrm{PGL}_{n}$ over $k^{s e p}$. Hence $G$ is an inner form of $\mathrm{PGL}_{n}$. Since $\mathrm{PGL}_{n}$ is split, $G=\mathrm{PGL}_{1}(A)$ is of inner type. The inner twisted forms arising from $G=\mathrm{PGL}_{1}(A)$ can be described very explicitly (see 20, Section 5 ). One of these inner twisted forms is the generalized Brauer-Severi variety. So let $m \leq n$. The generalized Brauer-Severi variety $\operatorname{BS}(m, A)$ is defined to be the subset of $\operatorname{Grass}_{k}(m n, A)$ consisting of those subspaces of $A$ which are right ideals of dimension $m \cdot n$ (see [18 or [5]). Recall that $\operatorname{Grass}_{k}(m n, A)$ is given the structure of a projective variety via the Plücker embedding (see [5])

$$
\operatorname{Grass}_{k}(m n, A) \longrightarrow \mathbb{P}\left(\wedge^{m n}(A)\right) .
$$

This gives an embedding $\operatorname{BS}(m, A) \rightarrow \mathbb{P}\left(\wedge^{m n}(A)\right)$ and a very ample line bundle $\mathcal{M}$. Note that for any $\operatorname{BS}(m, A)$ there exists a finite Galois field extension $E$ such that $\operatorname{BS}(m, A) \otimes_{k}$ $E \simeq \operatorname{Grass}_{E}\left(m n, n^{2}\right) \simeq \operatorname{Grass}_{E}(m, n)$. The Picard group $\operatorname{Pic}\left(\operatorname{Grass}_{E}(m, n)\right)$ is isomorphic to $\mathbb{Z}$ and has ample generator $\mathcal{O}(1) \simeq \operatorname{det}(\mathcal{Q})$ with $\mathcal{Q}$ being the universal quotient bundle on $\operatorname{Grass}_{E}(m, n)$. Recall that $\operatorname{Pic}(\operatorname{BS}(m, A)) \simeq \mathbb{Z}$ and that there is a positive generator $\mathcal{L}$ such that $\mathcal{L} \otimes_{k} E \simeq \mathcal{O}(r)$ for a suitable $r>0$. Since $\operatorname{Pic}(\operatorname{BS}(m, A))$ is cyclic, we have $\mathcal{L}^{\otimes s} \simeq \mathcal{M}$ for a suitable $s>0$. Therefore, $\mathcal{L}$ is ample. From the definition of $\operatorname{BS}(m, A)$ it is clear that $\mathcal{L}$ is also very ample.

Proposition 1. Let $X=\mathrm{BS}(m, A)$ be a genaralized Brauer-Severi variety over a field $k$ of characteristic zero and denote by $\mathcal{L}$ the very ample generator of $\operatorname{Pic}(X)$. Then $\left(X, \mathcal{L}^{\otimes d}\right)$ carries an Ulrich bundle for all $d \geq 1$. 
Proof. There is a finite Galois field extension $E$ of $k$ such that $X \otimes_{k} E$ is isomorphic to the Grassmannian $\operatorname{Grass}_{E}\left(m n, n^{2}\right)$. Consider the projection map $\pi: X \otimes_{k} E \rightarrow X$. Note that $\pi$ is finite and surjective. Let $k^{s e p}$ be a separable closure containing $E$. According to [11, Theorem 3.6 there is an Ulrich bundle $\mathcal{F}$ on $X \otimes_{k} k^{s e p} \simeq \operatorname{Grass}_{k^{s e p}}\left(m n, n^{2}\right)$ if it is embedded via the Plücker embedding, or equivalently, if it is embedded via $\mathcal{O}_{\operatorname{Grass}_{k} \operatorname{sep}\left(m n, n^{2}\right)}(1)$. This Ulrich bundle is obtained in the following way: Let

$$
0 \longrightarrow \mathcal{S}^{\vee} \longrightarrow \mathcal{O}_{\operatorname{Grass}_{k} \operatorname{sep}\left(m n, n^{2}\right)}^{\oplus n^{2}} \longrightarrow \mathcal{Q} \longrightarrow 0
$$

be the universal exact sequence. Then $\mathcal{F}$ is given by $\Sigma^{\lambda}\left(\mathcal{S}^{\vee}\right) \otimes \Sigma^{\beta} \mathcal{Q}$ for suitable partitions $\lambda$ and $\beta$. Since $\mathcal{S}^{\vee}$ and $\mathcal{Q}$ are also defined on $X \otimes_{k} E \simeq \operatorname{Grass}_{E}\left(m n, n^{2}\right)=: \mathbb{G}$ and since the Schur functor $\Sigma$ commutes with base change, the vector bundle $\mathcal{F}$ is also defined over $\mathbb{G}$. In other words, there is a bundle $\mathcal{F}^{\prime}$ on $\mathbb{G}$ such that $\mathcal{F}^{\prime} \otimes_{E} k^{\text {sep }} \simeq \mathcal{F}$. If we embed $\mathbb{G}$ via the Plücker embedding, i.e via $\mathcal{O}_{\mathbb{G}}(1)$, we get $H^{i}\left(X, \mathcal{F}^{\prime}(-l)\right)=0$ for any $i \in \mathbb{Z}$ and $1 \leq l \leq \operatorname{dim}(X)$. In fact, this follows from base change to $k^{s e p}$. Therefore, $\mathcal{F}^{\prime}$ is an Ulrich bundle for $\left(\mathbb{G}, \mathcal{O}_{\mathbb{G}}(1)\right)$. Now we use 4 , Corollary 3.2 to see that $\left(\mathbb{G}, \mathcal{O}_{\mathbb{G}}(d)\right)$ carries an Ulrich bundle for all $d>1$. Note that there is a line bundle $\mathcal{O}_{\mathbb{G}}(r)$ on $\mathbb{G}$ such that $\mathcal{L} \otimes_{k} E \simeq \mathcal{O}_{\mathbb{G}}(r)$. Since there is an Ulrich bundle $\mathcal{E}$ for $\left(\mathbb{G}, \mathcal{O}_{\mathbb{G}}(r d)\right)$ for all $d \geq 1$, we can apply [4, (3.6) to conclude that $\pi_{*} \mathcal{E}$ is an Ulrich bundle for $\left(X, \mathcal{L}^{\otimes d}\right)$.

Remark 2. The twisted Grassmannian $\operatorname{BS}(m, A)$ embedded into projective space by the very ample generator $\mathcal{L}$ cannot carry an Ulrich line bundle. Recall that $k^{\text {sep }}$ splits $A$, i.e $A \otimes_{k} k^{s e p} \simeq M_{n}\left(k^{s e p}\right)$. In fact, if $\mathcal{G}$ is an Ulrich line bundle, the base change $\mathcal{G} \otimes_{k} k^{\text {sep }}$ would give an Ulrich line bundle on $\mathbb{G}:=\operatorname{Grass}_{k} \operatorname{sep}\left(m n, n^{2}\right)$ with respect to $\mathcal{L} \otimes_{k} k^{s e p} \simeq \mathcal{O}_{\mathbb{G}}(r)$ for a suitable $r>1$. From [9], Proposition 2.1 we conclude that the degree of $\mathbb{G}$ is $>1$. Since Pic $(\mathbb{G})$ is generated by $\mathcal{O}_{\mathbb{G}}(1)$, there cannot exist an Ulrich bundle of rank one (see 4], Section 4 for an explanation). So, as in the case of ordinary Brauer-Severi varieties, there are no Ulrich line bundles on generalized ones.

Remark 3. Proposition 1 from above concerns the existence of Ulrich bundles on $\mathrm{BS}(m, A)$. It would be interesting to relate the minimal rank of such a bundle to the invariants period and index of $A$. In the case of ordinary Brauer-Severi varieties, i.e $m=1$ this is done in 21. Moreover, we wonder whether the obtained Ulrich bundles from Proposition 1 remain Ulrich in positive characteristic.

In general, the inner twisted flags arising from $G=\operatorname{PGL}_{1}(A)$, where $A$ is a central simple algebra of degree $n$, are varieties denoted by $\operatorname{BS}\left(n_{1}, \ldots, n_{l}, A\right), n_{1}<\cdots<n_{l}<n$, satisfying $\operatorname{BS}\left(n_{1}, \ldots, n_{l}, A\right) \otimes_{k} k^{s} \simeq \operatorname{Flag}_{k^{s}}\left(n_{1}, \ldots, n_{l} ; n\right)$. These partial twisted flags parametrize sequences $I_{1} \subseteq \cdots \subset I_{l} \subseteq A$ of right ideals with $\operatorname{dim}\left(I_{j}\right)=n \cdot n_{j}$, for $j=1, \ldots, l$ (see [20], Section 5). If $E$ is a splitting field of $A$, i.e $A \otimes_{k} E \simeq M_{n}(E)$, one has $\operatorname{BS}\left(n_{1}, \ldots, n_{l}, A\right) \otimes_{k}$ $E \simeq \operatorname{Flag}_{E}\left(n_{1}, \ldots, n_{l} ; n\right)$. For details, we refer to [18] and [20. Recall that a flag $\mathbb{F}_{L}:=$ $\operatorname{Flag}_{L}\left(n_{1}, \ldots, n_{l} ; n\right)$ over a field $L$ has $l$ projections $p_{i}: \operatorname{Flag}_{L}\left(n_{1}, \ldots, n_{l} ; n\right) \longrightarrow \operatorname{Grass}_{L}\left(l_{i}, n\right)$. The Picard group of $\mathbb{F}_{L}$ is generated by $\mathcal{L}_{i}=p_{i}^{*} \mathcal{O}_{\operatorname{Grass}_{L}\left(l_{i}, n\right)}(1)$. A line bundle $\mathcal{R}$ on $\mathbb{F}_{L}$ is ample if and only if $\mathcal{R}=\mathcal{L}_{1}^{\otimes a_{1}} \otimes \cdots \otimes \mathcal{L}_{l}^{\otimes a_{l}}$ with $a_{i}>0$. We set $\mathcal{O}_{\mathbb{F}_{L}}(1)=\mathcal{L}_{1} \otimes \cdots \otimes \mathcal{L}_{l}$.

Lemma 4. Let $X=\operatorname{BS}\left(n_{1}, \ldots, n_{l}, A\right)$ be a twisted flag as above and $E$ a finite splitting field of $A$. Then there is a very ample line bundle $\mathcal{L}$ on $X$ such that $\mathcal{L} \otimes_{k} E \simeq \mathcal{O}_{\mathbb{F}_{E}}(r)$ for a suitable $r>0$.

Proof. We give an elementary proof. A more structural argument is given in Proposition 7 below. The twisted flag $X$ has $l$ projections to generalized Brauer-Severi varieties

$$
\pi_{i}: \mathrm{BS}\left(n_{1}, \ldots, n_{l}, A\right) \longrightarrow \mathrm{BS}\left(l_{i}, A\right) .
$$

Let us denote by $\mathcal{O}_{\mathrm{BS}\left(l_{i}, A\right)}(1)$ the ample generators of $\operatorname{Pic}\left(\operatorname{BS}\left(l_{i}, A\right)\right)$. The line bundles $\mathcal{M}_{i}=\pi_{i}^{*} \mathcal{O}_{\mathrm{BS}\left(l_{i}, A\right)}(1)$ are in $\operatorname{Pic}(X)$ and so are the bundles

$$
\mathcal{M}_{1}^{\otimes a_{1}} \otimes \cdots \otimes \mathcal{M}_{l}^{\otimes a_{l}}
$$


for $a_{i} \in \mathbb{Z}$. Note that after base change to the splitting field $E$, we have $\mathcal{M}_{i} \otimes_{k} E \simeq \mathcal{L}_{i}^{\otimes b_{i}}$ for suitable positive $b_{i} \in \mathbb{Z}$. Therefore, we get

$$
\left(\mathcal{M}_{1} \otimes \cdots \otimes \mathcal{M}_{l}\right) \otimes_{k} E \simeq \mathcal{L}_{1}^{\otimes b_{1}} \otimes \cdots \otimes \mathcal{L}_{l}^{\otimes b_{l}} .
$$

Let $m$ be the least common multiple of all the $b_{i}$. By definition, there are positive integers $c_{i}$ such that $c_{i} \cdot b_{i}=m$. We set $\mathcal{N}=\mathcal{M}_{1}^{\otimes c_{1}} \otimes \cdots \otimes \mathcal{M}_{l}^{\otimes c_{l}}$ and obtain

$$
\mathcal{N} \otimes_{k} E \simeq \mathcal{L}_{1}^{\otimes b_{1} \cdot c_{1}} \otimes \cdots \otimes \mathcal{L}_{l}^{\otimes b_{l} \cdot c_{l}} \simeq \mathcal{O}_{\mathbb{F}_{E}}(m) .
$$

Since $\mathcal{O}_{\mathbb{F}_{E}}(m)$ is ample on $\mathbb{F}_{E}$, we get that $\mathcal{N}$ must be ample on $\operatorname{BS}\left(n_{1}, \ldots, n_{l}, A\right)$. We take $\mathcal{L}$ to be $\mathcal{N}^{\otimes s}$ for a suitable large integer $s>0$. In fact, there are plenty of such very ample line bundles. This completes the proof.

Proposition 5. Let $A$ be a degree $n$ central simple algebra over a field $k$ of characteristic zero and $E$ a finite splitting field. Let $X$ be one of the following twisted flag varieties:

$\mathrm{BS}(1, n-1, A), \quad \mathrm{BS}(1, n-2, A), \quad \mathrm{BS}(2, n-2, A), \quad \mathrm{BS}(m, m+1, A), \quad \operatorname{BS}(m, m+2, A)$.

Then there are very ample line bundles $\mathcal{L}$ on $X$ satisfying $\mathcal{L} \otimes_{k} E \simeq \mathcal{O}_{\mathbb{F}_{E}}(r)$ for suitable $r>0$. Moreover, for every such very ample line bundle $\left(X, \mathcal{L}^{\otimes d}\right)$ carries an Ulrich bundle for all $d \geq 1$.

Proof. The existence of very ample line bundles $\mathcal{L}$ satisfying the desired property follows from Lemma 4 . After base change to some splitting field $L$ of $A$, the variety $X$ becomes one of the following flags:

$$
\begin{aligned}
\operatorname{Flag}_{L}(1, n-1 ; n), \operatorname{Flag}_{L}(1, n-2 ; n), & \operatorname{Flag}_{L}(2, n-2 ; n), \\
& \operatorname{Flag}_{L}(m, m+1 ; n), \\
& \operatorname{Flag}_{L}(m, m+2 ; n) .
\end{aligned}
$$

We consider the projection map $\pi: X \otimes_{k} E \rightarrow X$ which is finite and surjective. Let $k^{s e p}$ be a separable closure containing $E$. Note that $k^{\text {sep }}$ is also a splitting field of $A$. According to [9], there is an Ulrich bundle on $X \otimes_{k} k^{\text {sep }}$ with respect to $\mathcal{O}_{X \otimes_{k} k^{s e p}}(1)$. This Ulrich bundle is obtained in the following way: Let $0=\mathcal{T}_{0} \subset \mathcal{T}_{1} \subset \mathcal{T}_{2} \subset \mathcal{T}_{3}$ be the collection of tautological subbundles on $X \otimes_{k} k^{\text {sep }}$ and $\mathcal{U}_{i}=\mathcal{T}_{i} / \mathcal{T}_{i-1}$. Then $\Sigma^{\lambda}\left(\mathcal{U}_{1}^{\vee}\right) \otimes \Sigma^{\alpha}\left(\mathcal{U}_{2}^{\vee}\right) \otimes \Sigma^{\beta}\left(\mathcal{U}_{3}^{\vee}\right)$ is an Ulrich bundle for suitable partitions $\lambda, \alpha$ and $\beta$. These bundles can also be defined over $\mathbb{F}_{E}=X \otimes_{k} E$. We argue as in the proof of Proposition 1 to conclude that there is an Ulrich bundle on $\mathbb{F}_{E}$ with respect to $\mathcal{O}_{\mathbb{F}_{E}}(1)$. Again, we use 4, Corollary 3.2 to see that $\left(\mathbb{F}_{E}, \mathcal{O}_{\mathbb{F}_{E}}(d)\right)$ carries an Ulrich bundle for all $d>1$. Now take a very ample line bundle $\mathcal{L}$ on $\mathbb{F}_{E}$ satisfying $\mathcal{L} \otimes_{k} E \simeq \mathcal{O}_{\mathbb{F}_{E}}(r)$ for some $r>0$. Since there is an Ulrich bundle $\mathcal{E}$ for $\left(\mathbb{F}, \mathcal{O}_{\mathbb{F}_{E}}(r d)\right)$ for all $d \geq 0$, we can apply [4, (3.6) to conclude that $\pi_{*} \mathcal{E}$ is an Ulrich bundle for $\left(X, \mathcal{L}^{\otimes d}\right)$. This proves the assertion.

Theorem 6 (Type $A_{n}$ ). Let $A$ be a degree $n$ central simple algebra over a field $k$ of characteristic zero and $E$ a finite splitting field. Let $X$ one of the following twisted flags:

$$
\begin{aligned}
& \mathrm{BS}(m, A), \mathrm{BS}(1, n-1, A), \quad \mathrm{BS}(1, n-2, A), \quad \mathrm{BS}(2, n-2, A), \quad \mathrm{BS}(m, m+1, A), \\
& \mathrm{BS}(m, m+2, A) \text {. }
\end{aligned}
$$

Then there are very ample line bundles $\mathcal{L}$ on $X$ satisfying $\mathcal{L} \otimes_{k} E \simeq \mathcal{O}_{\mathbb{F}_{E}}(r)$ for suitable $r>0$. Moreover, for every such very ample bundle $\left(X, \mathcal{L}^{\otimes d}\right)$ carries an Ulrich bundle for all $d \geq 1$.

Proposition 7. Let $X$ be a smooth projective geometrically integral variety over a field $k$. Assume $X \otimes_{k} k^{s e p}$ is embedded into projective space via $\mathcal{O}_{X \otimes_{k} k^{s e p}}(1)$, i.e $\mathcal{O}_{X \otimes_{k} k^{s e p}}(1)=$ $i^{*} \mathcal{O}_{\mathbb{P}^{N}}(1)$ for an embedding $i: X \otimes_{k} k^{\text {sep }} \rightarrow \mathbb{P}^{N}$. Then there is a very ample line bundle $\mathcal{L}$ on $X$ satisfying $\mathcal{L} \otimes_{k} k^{\text {sep }} \simeq \mathcal{O}_{X \otimes_{k} k^{s e p}}(r)$ for a suitable $r>0$. 
Proof. Let $G$ be the absolute Galois group. It is well know that there is an exact sequence arising from the Leray spectral sequence

$$
0 \longrightarrow \operatorname{Pic}(X) \longrightarrow \operatorname{Pic}\left(X \otimes_{k} k^{s e p}\right)^{G} \stackrel{\delta}{\longrightarrow} \operatorname{Br}(k) \longrightarrow \operatorname{Br}(k(X)) .
$$

Note that every element of $\operatorname{Br}(k)$ has finite order. Now let $\mathcal{O}_{X \otimes_{k} k^{s e p}}(m)$ with $m>0$ be an element of the cokernel of $\operatorname{Pic}(X) \rightarrow \operatorname{Pic}\left(X \otimes_{k} k^{s e p}\right)^{G}$. If this element is trivial, we are done. Assume $\mathcal{O}_{X \otimes_{k} k^{\text {sep }}}(m)$ is non-trivial. Then $\delta\left(\mathcal{O}_{X \otimes_{k} k^{\text {sep }}}(m)\right)$ is a non-trivial Brauer-equivalence class $[B] \in \operatorname{Br}(k)$. If $d>0$ is the order of $[B]$ in $\operatorname{Br}(k)$, we obtain that $\delta\left(\mathcal{O}_{X \otimes_{k} k^{s e p}}(m d)\right)=[k]$. This implies that there exists a line bundle $\mathcal{L}$ on $X$ such that $\mathcal{L} \otimes_{k} k^{s e p} \simeq \mathcal{O}_{X \otimes_{k} k^{s e p}}(m d)$. Since $\mathcal{O}_{X \otimes_{k} k^{s e p}}(m d)$ is very ample, we conclude that $\mathcal{L}$ must be very ample. Therefore, there is a very ample line bundle $\mathcal{L}$ on $X$ satisfying $\mathcal{L} \otimes_{k} k^{s e p} \simeq \mathcal{O}_{X \otimes_{k} k^{s e p}}(r)$ for a suitable $r>0$.

Let $Y$ be one of the following flags: symplectic Grassmannians $\operatorname{IGrass}_{k} \operatorname{sep}(2,2 n)$ for $n \geq 2$, odd and even-dimensional quadrics, orthogonal Grassmannians $\operatorname{OGras}_{k} \operatorname{sep}(2, m)$ for $m \geq 4$, OGrass $k_{\text {sep }}(3,4 q+6)$ for $q \geq 0$ and $\operatorname{OGrass}_{k}$ sep $(4,8)$. Then $\operatorname{Pic}(Y) \simeq \mathbb{Z}$ is generated by a very ample line bundle (see 16, Section 2$)$. We will denote this very ample generator by $\mathcal{O}_{Y}(1)$. For inner twisted forms of $Y$, we refer to [20], Section 5 .

Theorem 8 (Type $B_{n}, C_{n}$ and $D_{n}$ ). Let $X$ be an inner twisted form of $Y$ from above. Then there are very ample line bundles $\mathcal{L}$ on $X$ satisfying $\mathcal{L} \otimes_{k} k^{\text {sep }} \simeq \mathcal{O}_{Y}(r)$ for suitable $r>0$. Moreover, for every such very ample bundle $\left(X, \mathcal{L}^{\otimes d}\right)$ carries an Ulrich bundle for all $d \geq 1$.

Proof. The existence of very ample line bundles $\mathcal{L}$ satisfying the desired property follows from Proposition 7. Note that for every inner twisted flag from above, there is a finite field extension $E$ of $k$ such that $X \otimes_{k} E$ is isomorphic to the corresponding flag. We prove the statement only for the case $X=\operatorname{IGrass}_{k} \operatorname{sep}(2,2 n)$ for $n \geq 2$, because the proof for the remaining cases is analogous. So let $E$ be a finite splitting field contained in $k^{s e p}$, i.e $X \otimes_{k} E \simeq \operatorname{IGrass}_{E}(2,2 n)$. The main theorem of [16] states that the listed flags carry Ulrich bundles over $k^{s e p}$ with respect to $\mathcal{O}_{Y}(1)$. In particular, $Y:=\operatorname{IGrass}_{k^{s e p}}(2,2 n)$ carries an Ulrich bundle $\mathcal{E}$ with respect to $\mathcal{O}_{Y}(1)$ which is given as $\Sigma^{(2 n-3,0, \ldots, 0)}\left(\mathcal{U}^{\vee}\right)$ with $\mathcal{U}$ being the tautological subbundle (see [16], Corollary 3.6). This vector bundle can also defined over $\operatorname{IGrass}_{E}(2,2 n)$, i.e there is a vector bundle $\mathcal{E}^{\prime}$ on $\operatorname{IGrass}_{E}(2,2 n)$ such that $\mathcal{E}^{\prime} \otimes_{E} k^{s e p} \simeq \mathcal{E}$. We argue as in Proposition 1 to conclude that $\mathcal{E}^{\prime}$ is an Ulrich bundle on $\operatorname{IGrass}_{E}(2,2 n)$ with respect to $\mathcal{O}_{\operatorname{IGrass}_{E}(2,2 n)}(1)$. We use 4], Corollary 3.2 to see that $\left.\operatorname{IGrass}_{E}(2,2 n), \mathcal{O}_{\text {IGrass }_{E}(2,2 n)}(d)\right)$ carries an Ulrich bundle for all $d>1$. From Proposition 7 we can conclude that there is a very ample line bundle $\mathcal{L}$ on $X$ satisfying $\mathcal{L} \otimes_{k} E \simeq \mathcal{O}_{\text {IGrass }_{E}(2,2 n)}(r)$ for a suitable $r>0$. As in Propositions 1 and 5 we use the projection $\pi: X \otimes_{k} E \rightarrow X$, which is finite and surjective, to produce an Ulrich bundle for $\left(X, \mathcal{L}^{\otimes d}\right)$ for all $d \geq 1$.

Let $Y$ be one of the flags from above. Then $\operatorname{Pic}(Y) \simeq \mathbb{Z}$ is generated by the very ample line bundle $\mathcal{O}_{Y}(1)$. For inner twisted forms $X$ of $Y$, this implies $\operatorname{Pic}(X) \simeq \mathbb{Z}$. The ample generator $\mathcal{L}$ of $\operatorname{Pic}(X)$ obviously satisfies $\mathcal{L} \otimes_{k} k^{\text {sep }} \simeq \mathcal{O}_{Y}(r)$ for a suitable $r>0$. Moreover, the ample generator of $\operatorname{Pic}(X)$ is also very ample.

Corollary 9. Let $X$ be as in Theorem 8 and denote by $\mathcal{L}$ the very ample generator of $\operatorname{Pic}(X)$. Then $\left(X, \mathcal{L}^{\otimes d}\right)$ carries an Ulrich bundle for all $d \geq 1$.

Acknowledgement. I thank Nikita Karpenko, Daniel Krashen and Kirill Zainoulline for answering questions concerning twisted flags. This research was conducted in the framework of the research training group GRK 2240: Algebro-geometric Methods in Algebra, Arithmetic and Topology, which is funded by the DFG. 


\section{REFERENCES}

[1] M. Aprodu, L. Costa and R.M. Miró-Roig: Ulrich bundles on ruled surfaces, arXiv:1609.08340 1 (2016).

[2] M. Aprodu, G. Farkas and A. Ortega: Minimal resolutions, Chow formsand Ulrich bundles on K3 surfaces, J. Reine. Angew. Math. to appear.

[3] A. Beauville: Ulrich bundles on abelian surfaces, Proc. Amer. Math. Soc. 144 (2016), 46094611.

[4] A. Beauville: An introduction to Ulrich bundles, European J. Math 4 (2018), 26-36.

[5] A. Blanchet: Function fields of generalized Brauer-Severi varieties, Communications in Alg. 19 (1991), 97-118.

[6] J.P. Brennan, J. Herzog and Bernd Ulrich: Maximally generated Cohen-Macaulay modules, Math. Scand. 61 (1987), 181-203.

[7] J.P. Brennan, J. Herzog and Bernd Ulrich: Linear maximal Cohen-Macaulay modules over strict complete intersections, J. Pure Appl. Algebra 71 (1991), 187-202.

[8] L. Borisov and H. Nuer: Ulrich bundles on Enriques surfaces, arXiv:1606.01459v1 (2016).

[9] I. Coskun, J. Huizenga, R.M. Miró-Roig and M. Woolf: Ulrich Schur bundles on Flag varieties, arXiv:1512.06193v1 [math.AG] (2015).

[10] L. Costa, R.M. Miró-Roig and J. Pons-Llopis: The representation type of Segre varieties, Adv. Math. 230 (2012), 1995-2013.

[11] L. Costa and R.M. Miró-Roig: GL( $V)$-invariant Ulrich bundles on Grassmannians, Math. Ann. 361 (2015), 443-457.

[12] L. Costa and R.M. Miró-Roig: Cohomological characterization of vector bundles on multiprojective spaces, J. Algebra 294 (2005), 73-96.

[13] D. Eisenbud, F.O. Schreyer and J. Weyman: Resultants and Chow forms via exterior syzigies, Amer. Math. Soc. 16 (2003), 537-579.

[14] D. Faenzi: Ulrich bundles on K3 surfaces, arXiv:1807.07826 v1 [math.AG] (2018).

[15] M.L. Fania, M. Lelli-Chiesa and J. Pons-Llopis: Ulrich bundles on three dimensional scrolls, arXiv:1808.00074 1 [math.AG] (2018).

[16] A. Fonarev: Irreducible Ulrich bundles on isotropic Grassmannians. Mosc. Math. J. 16 (2016), 711-726.

[17] D. Hanes: Special conditions on maximal Cohen-Macaulay modules, and applications to the theory of multiplicities, University of Michigan Ph.D. thesis (1999).

[18] M-A. Knus, A. Merkurjev, M. Rost and J-P. Tignol: The Book of Involutions. AMS Coll. Publ. 44, AMS, Providence, RI (1998).

[19] R.M. Miró-Roig: The representation type of rational normal scrolls, Rend. Circ.Mat. Palermo 62 (2013), 153-164.

[20] A.S. Merkurjev, I.A. Panin and A.R. Wadsworth: Index reduction formulas for twisted flag varieties, I K-Theory 10, (1996), 517-596.

[21] S. Novaković: Ulrich bundles on Brauer-Severi varieties, arXiv:1807.10919 [math.AG] (2018).

MATHEMATISCHES INSTITUT, HEINRICH-HEINE-UNIVERSITÄT 40225 DÜSSELDORF, GERMANY

E-mail adress: novakovic@math.uni-duesseldorf.de 